\title{
Relationships between technological and nutritional meat quality parameters in local poultry populations (Gallus gallus) of Benin
}

\author{
P.U. TOUGAN ${ }^{1}$, M. DAHOUDA ${ }^{2}$, C.F.A. SALIFOU ${ }^{1}$, G.S. AHOUNOU ${ }^{1}$, D.N.F. KOSSOU ${ }^{1}$, \\ C. AMENOU ${ }^{1}$, C.E. KOGBETO ${ }^{1}$, M.T. KPODEKON ${ }^{1}$, G.A. MENSAH ${ }^{3}$, G. LOGNAY ${ }^{4}$, \\ A. THEWIS ${ }^{5}$ and I.A.K. YOUSSAO ${ }^{1 *}$ \\ ${ }^{I}$ Department of Animal Production and Health, Polytechnic School of Abomey-Calavi, \\ 01 BP 2009, Cotonou, Republic of Benin. \\ ${ }^{2}$ Department of Animal Production, Faculty of Agronomic Science, University of Abomey-Calavi, \\ 01 BP 526, Republic of Benin. \\ ${ }^{3}$ Agricultural Research Center of Agonkanmey, National Institute of Agricultural Research of Benin, \\ 01 BP 884, Cotonou 01, Republic of Benin. \\ ${ }^{4}$ Laboratory of Analytical Chemistry, Gembloux Agro-Bio Tech, University of Liège, 2, \\ Passage des Déportés -5030 Gembloux, Belgique. \\ ${ }^{5}$ Animal Sciences Unit, Gembloux Agro-Bio Tech, University of Liege, Passage des Déportés, 2, \\ 5030 Gembloux, Belgium. \\ *Corresponding author, E-mail: iyoussao@yahoo.fr, issaka.youssao@epac.uac.bj; Tel :00 22995285988 , \\ 0022997912074, Fax : 0022921360199
}

\begin{abstract}
The current work aims at determining relationships between technological and nutritional meat quality parameters in Holli, Fulani, Sahoue, North and South indigenous chicken ecotypes of Benin. Color parameters $\left(\mathrm{L}^{*}, \mathrm{a}^{*}, \mathrm{~b}^{*}\right.$, hue and chroma), $\mathrm{pH}$, texture, dry matter content, protein content, fat content, and ash content were collected on 52 cockerels of each ecotype slaughtered at 24 weeks old. In Holli chickens, dry matter content was highly and positively correlated with protein content $(\mathrm{P}<0.001 ; \mathrm{r}=0.57)$, moderately and positively associated to ash content, redness, yellowness and chroma $(\mathrm{P}<0.01)$, but weakly and negatively correlated with the luminance and hue $(\mathrm{P}<0.05)$. Fat content was highly and positively correlated with the texture, $\mathrm{pH} 4, \mathrm{pH} 12$, $\mathrm{pH} 24, \mathrm{a}^{*}$ and chroma $(\mathrm{P}<0.001 ; 0.46 \leq \mathrm{r} \leq 0.85)$, but highly and negatively correlated with the protein content and luminance $(\mathrm{P}<0.001 ;-0.64 \leq \mathrm{r} \leq-0.47)$. Except dry matter content, protein content was negatively correlated with the others parameters. Correlations between dry matter and the other meat quality traits of North chickens were similar to those of Holli chickens except fat content, $\mathrm{pH}, \mathrm{L}^{*}, \mathrm{~b}^{*}$ and hue. Except texture, the correlations between dry matter concentration and the other meat quality traits of South and Fulani chickens were similar to those of Sahoue chickens. South chickens and to a lesser extent Fulani and Sahoue chickens were characterized by higher dry matter, shear force, breast cooking loss, yellowness and $\mathrm{pH} 24$, while Holli chickens were characterized by greater protein content, organic matter content, moisture, thigh cooking loss, hue, $\mathrm{pH} 1, \mathrm{pH} 4, \mathrm{pH} 8$ and $\mathrm{pH} 12$. North chickens were characterized by higher ash content, fat content and luminance.
\end{abstract}

() 2013 International Formulae Group. All rights reserved.

Keywords: Correlation, indigenous chicken, meat quality, principal components analysis. 


\section{INTRODUCTION}

In Benin, the indigenous chickens represent $81.3 \%$ of the national poultry flock (CountryStat, 2012) and are an important source of animal protein supply for the population and income for producers and poultry sellers (Tougan, 2008). Despite the importance of this poultry flock, local poultry meat production remains below the consumer demand. This shortage created pressure on every form of food supply and lead on increase of meat imports (2.5 times from 2000 to 2010 accordingly to CountryStat, 2012). Despite the low domestic production of local chickens $(2,020$ tons in 2010), local chicken meat is more appreciated by consumers in comparison with imported chicken meat because of its leanness and relatively lower purchasing price (Mankor, 2009). The lack of religious restriction against indigenous chicken consumption justifies the perennity of its production in Benin (Tougan, 2008).

The local population of poultry of the species Gallus gallus of Benin is composed of various ecotypes among which are North, South, Holli, Fulani or Peuhl and Sahoue ecotypes (Bonou, 2006). These indigenous poultry have a remarkable heterogeneity in phenotypical traits (Youssao et al., 2007) and polymorphism trait (Youssao et al., 2009). Several studies were carried out on carcass traits of these local genetic types (Youssao et al., 2009; Youssao et al., 2010; Youssao et al., 2012; Tougan et al., 2013a). The recent studies carried out on the effect of breeding mode, type of muscle and slaughter age on technological meat quality of these local chickens of Benin (Tougan et al., 2013c) on the one hand, and on variation of nutritional quality of meat of local poultry population of
Gallus gallus specie of Benin (Tougan et al., 2013d) on the other hand showed significant variation of technological and nutritional qualities.

Although the difference between the five ecotypes of local chicken is well known on technological and nutritional meat quality traits, no data exist on the correlations between their technological and nutritional qualities traits.

For accuracy and better judgment of technological and nutritional meat quality traits of indigenous chicken population of Benin, the current study aims at pointing out the relationships between the technological and nutritional meat quality traits of Holli, Fulani, Sahoue, North and South indigenous chicken ecotypes of Benin.

\section{MATERIALS AND METHODS Area of study}

The current study was conducted conjointly at the experimental farm of "Ecole Polytechnique d'Abomey-Calavi (EPAC)" and at the traditional poultry breeders located in Abomey-Calavi (latitude of $6^{\circ} 27^{\prime}$ north and at a longitude of $2^{\circ} 21^{\prime}$ east) in Atlantic Department from April 2011 to June 2012. The Commune of Abomey-Calavi covers an area of $650 \mathrm{~km}^{2}$ with a population of 307,745 inhabitants (INSAE, 2010).

\section{Birds sampling and characteristics of breeding systems}

The chickens used in this trial were produced from breeding nuclei of 10 hens and 3 cocks of each genetic type, reared in confinement at the experimental farm of EPAC as described by Tougan et al. (2013a). Two groups of 26 chickens of each ecotype 
were reared respectively under traditional free range and improved confinement breeding systems and then slaughtered at 24 weeks old.

In free range system, the birds were let scavenge during the day but housed at night in rudimentary shelters (traditional henhouse made of mud, straw or wicker), or kept outside on any support that could serve as a perch. The feeding is not rational and the birds fed themselves by gleaning, but, some grain supplement was distributed to birds occasionally. Their diet was composed of energetic elements (kitchen waste, bran ...), vitamins (green fodder, sprouted grains ...), minerals (salt and pounded shells) and protein from termites and leguminous plants. Water was distributed in rudimentary watering tank. Various discarded containers were often used for drinking. In this type of farming, neither health follow-up nor prophylactic standard were applied.

Concerning confinement breeding system, the birds were bred on a fresh wood shavings litter in buildings of california type. The livestock equipment used were composed of brooders, feeders, drinkers ... The number of these devices depended on the number of birds in the henhouse. All the animals were fed with the same diet. Three diets were used: starting (2880 ME Kcal/kg and $18 \%$ of crude protein), growing (2969 ME Kcal/kg and $18 \%$ crude protein) and laying (2800 ME Kcal/kg of feed and $20 \%$ of crude protein). The starter feed was used from the hatching to the age of 2 months and the growth feed from 2 month old to the point of laying (22 weeks). From the point of laying to the end of the experimentation, the laying feed was used. The animals were fed ad-libitum throughout the study. Feed transitions were done during three days between the different growth periods by gradual incorporation to the previous diet with the respective proportions of 25,50 and $75 \%$ of the new diet. The composition and the nutrient contents of each diet are given in Table 1. Habitat, Health and medical prophylaxis used in confinement breeding system were described by Tougan et al. (2013b).

\section{Slaughtering process and analytical methods}

The slaughtering process used in the current study is described by Tougan et al. (2013a). After slaughtering, the cuts of breast and thigh-drumstick were used to evaluate the technological properties $(\mathrm{pH}$, color, hue, chroma and texture) and chemical composition (moisture, protein, fat, and ash) of meat. The $\mathrm{pH}$, the color, the hue value, the chroma value and the texture was determined as described in previous study by Tougan et al. (2013c), while dry matter content, protein content, fat content, and ash content were determined as described by Tougan et al. (2013d). Those different parameters evaluated herein were the mean of the values recorded from both thigh and breast muscles.

\section{Statistical analysis}

The data collected on technological and nutritional meat quality parameters $(\mathrm{pH}$, color, hue, chroma, texture, dry matter content, protein content, fat content, and ash content) of the five genetic types of chicken were analyzed with Statistical Analysis System software (SAS 2006). The correlations between the different variables were determined by ecotype using Proc corr procedure of SAS (SAS 2006). Principal 
Components Analysis (PCA) of technological and nutritional meat quality parameters was carried out for each ecotype and for all ecotypes by the Proc princompt procedure of SAS (2006).

\section{RESULTS}

Correlations between technological and nutritional meat quality parameters

The correlations between technological and nutritional meat quality parameters were presented by ecotype.

The Table 2 presents on top of diagonal the correlations between technological and nutritional meat quality parameters for Holli ecotype. In Holli chickens, dry matter content was highly and positively correlated with protein content $(\mathrm{P}<0.001 ; \mathrm{r}=0.57)$, moderately and positively associated to ash content, redness $\left(a^{*}\right)$, yellowness $\left(b^{*}\right)$ and chroma value $(\mathrm{P}<0.01 ; 0.34 \leq \mathrm{r} \leq 0.37)$, weakly and positively associated to the $\mathrm{pH}$ recorded 1 hour post-mortem $(\mathrm{pH} 1)$ and 4 hours (pH4) post mortem $(\mathrm{P}<0.05 ; 0.27 \leq \mathrm{r} \leq$ 0.28 ), but weakly and negatively correlated with the luminance $\left(\mathrm{L}^{*}\right)$ and hue value $(\mathrm{P}<0.05 ;-0.3 \leq \mathrm{r} \leq-0.28)$. The ash concentration was weakly and positively associated to the fat content, shear force, $\mathrm{pH} 24, \mathrm{a}^{*}, \mathrm{~b}^{*}$ and chroma $(\mathrm{P}<0.05,0.27 \leq \mathrm{r} \leq$ 0.32 ), but weakly and negatively correlated with the luminance $(\mathrm{P}<0.05 ; \mathrm{r}=-0.27)$. Fat content was highly and positively correlated with the texture, extension, pH4, pH12 (recorded 12 hours post mortem), pH24 (recorded 24 hours post mortem), a* and chroma $(\mathrm{P}<0.001 ; 0.46 \leq \mathrm{r} \leq 0.85)$, moderately and positively associated to $\mathrm{pH} 1$ and yellowness $(\mathrm{P}<0.01 ; 0.34 \leq \mathrm{r} \leq 0.39)$, weakly and positively associated to the $\mathrm{pH} 8$
$(\mathrm{P}<0.05, \mathrm{r}=0.3)$, but highly and negatively correlated with the protein content and luminance $(\mathrm{P}<0.001 ;-0.64 \leq \mathrm{r} \leq-0.47)$. Except dry matter content, protein content was negatively correlated with the others technological and nutritional meat quality parameters with the high negative correlations found with the shear force and redness $(\mathrm{P}<0.001 ;-0.45 \leq \mathrm{r} \leq-0.44)$. Furthermore, shear force was highly and positively correlated with the extension, $\mathrm{a}^{*}$ and chroma value $(\mathrm{P}<0.001 ; 0.64 \leq \mathrm{r} \leq 0.73)$, but highly and negatively associated to the luminance $(\mathrm{P}<0.001 ; \mathrm{r}=-0.44)$. Similarly, high and significant relationships were also found between $\mathrm{pH} 1$ and $\mathrm{pH} 4, \mathrm{pH} 8, \mathrm{pH} 12, \mathrm{pH} 24$, redness and chroma value $(\mathrm{P}<0.001 ; 0.43 \leq \mathrm{r}$ $\leq 0.76)$. Nevertheless, the luminance was negatively associated to all meat quality parameters studied in current study except protein content. The redness was highly and negatively associated to protein content and luminance $(\mathrm{P}<0.001 ;-0.56 \leq \mathrm{r} \leq-0.45)$, but highly and positively correlated with the fat content, shear force, extension, $\mathrm{pH} 1$ and $\mathrm{pH} 4$ $(\mathrm{P}<0.001 ; 0.44 \leq \mathrm{r} \leq 0.85)$. Similar relationships were also observed between the chroma value and the others meat quality traits studied herein.

The correlations between technological and nutritional meat quality parameters for North ecotype are under the diagonal of the Table 2. The correlations between dry matter and the other meat quality traits of North chickens were similar to those of Holli chickens except fat content, $\mathrm{pH}, \mathrm{L}^{*}, \mathrm{~b}^{*}$ and hue value. However, ash concentration was weakly and positively associated to the $\mathrm{pH} 24$ $(\mathrm{P}<0.05 ; \mathrm{r}=0.33)$, but weakly and negatively correlated with the extension $(\mathrm{P}<0.05 ; \mathrm{r}=$ - 
0.30). Fat content was highly and positively correlated with the texture, $\mathrm{pH} 8, \mathrm{a}^{*}$ and hue $(\mathrm{P}<0.001 ; 0.46 \leq \mathrm{r} \leq 0.85)$, moderately and positively associated to $\mathrm{pH} 1, \mathrm{pH} 4$, and $\mathrm{pH} 24$ $(\mathrm{P}<0.01 ; 0.36 \leq \mathrm{r} \leq 0.43)$, weakly and positively associated to the extension and pH12 $(\mathrm{P}<0.05, \mathrm{r}=0.35)$, but highly and negatively correlated with the protein content and luminance $(\mathrm{P}<0.001 ;-0.78 \leq \mathrm{r} \leq-0.54)$. Except dry matter content and luminance, protein content was negatively correlated with the others technological and nutritional meat quality parameters with the high negative correlations found with the shear force, extension, redness, hue and chroma $(\mathrm{P}<0.001$; $-0.69 \leq \mathrm{r} \leq-0.46$ ). As in Holli chickens, shear force in North chicken was highly and positively correlated with the extension, and redness $(\mathrm{P}<0.001 ; 0.45 \leq \mathrm{r} \leq 0.75)$, but also moderately and positively associated to the chroma value $(\mathrm{P}<0.01 ; \mathrm{r}=0.42)$. The luminance was highly and negatively associated to the dry matter content, fat content, $\mathrm{pH} 8$ and $\mathrm{pH} 24(\mathrm{P}<0.001 ;-0.55 \leq \mathrm{r} \leq-$ 0.46 ), moderately and negatively correlated with pH1, pH4 and pH12 (P<0.01; $-0.44 \leq \mathrm{r} \leq$ $-0.43)$. The redness was highly and negatively associated to protein content and luminance $(\mathrm{P}<0.001 ;-0.69 \leq \mathrm{r} \leq-0.62)$, but highly and positively correlated with the fat content and shear force $(\mathrm{P}<0.001 ; 0.44 \leq \mathrm{r} \leq 0.85)$.

The Table 3 presents on the top of diagonal the correlations between technological and nutritional meat quality parameters for Fulani ecotype. The relationships between dry matter and the others quality traits in Fulani chickens were similar to those recorded in South chickens. Contrary to Sahoue and South chickens, ash content in Fulani chicken was weakly and negatively associated to the $\mathrm{pH} 4, \mathrm{pH} 8, \mathrm{pH} 12$ and $\mathrm{pH} 24(\mathrm{P}<0.05 ;-0.33 \leq \mathrm{r} \leq-0.29)$. Fat content was highly and positively correlated with the texture, extension, $\mathrm{a}^{*}$ and chroma $(\mathrm{P}<0.001 ; 0.54 \leq \mathrm{r} \leq 0.81)$, moderately and positively associated to $\mathrm{pH} 4, \mathrm{pH} 8 \mathrm{pH} 12$ and hue $(\mathrm{P}<0.01 ; 0.37 \leq \mathrm{r} \leq 0.39)$, weakly and positively associated to the $\mathrm{pH} 1$ and $\mathrm{pH} 24$ $(\mathrm{P}<0.05,0.3 \leq \mathrm{r} \leq 0.31)$, but highly and negatively correlated with the protein content and luminance $(\mathrm{P}<0.001 ;-0.68 \leq \mathrm{r} \leq-0.61)$ as found in the others genotypes. Except dry matter content, ash content and protein content, luminance was negatively associated to all meat quality parameters in Fulani chickens. Similarly, protein content was negatively correlated with the others technological and nutritional meat quality parameters except dry matter content, luminance and yellowness.

Correlations between technological and nutritional meat quality parameters for Sahoue ecotype are shown under the diagonal of Table 3. In Sahoue chickens, dry matter content was highly and positively correlated with yellowness $(\mathrm{P}<0.001 ; \mathrm{r}=0.55)$, moderately and positively associated to the fat content, protein content, and chroma $(\mathrm{P}<0.01$; $0.37 \leq \mathrm{r} \leq 0.43)$, weakly and positively associated to the redness $(\mathrm{P}<0.05, \mathrm{r}=0.35)$. Fat content was highly and positively correlated with the texture, $a^{*}$, extension and chroma $(\mathrm{P}<0.001 ; 0.54 \leq \mathrm{r} \leq 0.81)$, moderately and positively associated to dry matter content, $\mathrm{pH} 4, \mathrm{pH} 8$ and $\mathrm{pH} 12$ ( $\mathrm{P}<0.01$; $0.37 \leq \mathrm{r} \leq 0.39$ ), weakly and positively associated to the $\mathrm{pH} 1$ and $\mathrm{pH} 24(\mathrm{P}<0.05,0.30$ $\leq \mathrm{r} \leq 0.31$ ), but highly and negatively correlated with the protein content and luminance $(\mathrm{P}<0.001 ;-0.68 \leq \mathrm{r} \leq 0.61)$ as 
found in the others genotypes. Except dry matter content and luminance and yellowness, protein content was negatively correlated with the others technological and nutritional meat quality parameters. Furthermore, shear force was highly and positively correlated with the fat content, extension, $\mathrm{a}^{*}$ and chroma value $(\mathrm{P}<0.001 ; 0.46 \leq \mathrm{r} \leq 0.81)$, moderately and positively associated to the $\mathrm{pH} 4$ and hue $(\mathrm{P}<0.01 ; 0.4 \leq \mathrm{r} \leq 0.42)$, weakly and positively associated to the yellowness, but weakly and negatively associated to the luminance and protein content $(\mathrm{P}<0.05 ;-0.79$ $\leq \mathrm{r} \leq 0.68)$.

Correlations between technological and nutritional meat quality parameters for South ecotype are showed in Table 4. Except shear force and extension, the correlations between dry matter concentration and the other meat quality traits of South chickens were similar to those of Sahoue chickens. However, the correlations between ash content and the other meat quality in South ecotype were not significant. The correlations between fat content and the other meat quality in South ecotype were comparable to those of North chicken. However, protein content was highly and positively associated to dry matter content and luminance $(\mathrm{P}<0.001 ; 0.44 \leq \mathrm{r} \leq 0.53)$, but negatively correlated with the shear force, redness and chroma value $(\mathrm{P}<0.001 ;-0.6 \leq \mathrm{r} \leq$ -0.52). The shear force was highly and positively correlated with $\mathrm{a}^{*}$ and chroma value $(\mathrm{P}<0.001 ; 0.53 \leq \mathrm{r} \leq 0.59)$, but moderately and negatively associated to the luminance $(\mathrm{P}<0.01 ; \mathrm{r}=-0.42)$.

Overall, fat content was more associated with the other meat quality traits in chicken of all ecotypes studied. Furthermore, technological meat quality parameters were more associated to nutritional meat quality parameters in Fulani and Holli, chickens than Sahoue, South and North chickens.

\section{Principal components analysis of technological and nutritional meat quality parameters}

The Figure 1 presents the principal components analysis of technological and nutritional meat quality parameters of the five genetic types of chicken studied. Indeed, principal components analysis of technological and nutritional meat quality parameters discriminated the five ecotypes according to their meat quality. The first axis explains $46.09 \%$ of the variation and opposed meat quality traits of Holli chickens to those of South chickens and to a lesser extent to Fulani and Sahoue chickens. The second axis explains $35.54 \%$ of the variation and opposed technological and nutritional meat quality parameters of North chickens to those of Holli and South chickens.

South chickens and to a lesser extent Fulani and Sahoue chickens were characterized by higher moisture, shear force, breast cooking loss, yellowness, extension and pH24, while Holli chickens were characterized by greater protein content, organic matter content, dry mater, thigh cooking loss, hue value, $\mathrm{pH} 1, \mathrm{pH} 4, \mathrm{pH} 8$ and pH12. North chickens were characterized by higher ash content, fat content and luminance. 
P.U. TOUGAN et al. / Int. J. Biol. Chem. Sci. 7(6): 2450-2467, 2013

Table 1: Composition and nutrient content of the starting, growing and laying diets.

\begin{tabular}{lccc}
\hline Composition & Starting diet & Growing diet & Laying diet \\
\hline Soy cakes $(\mathrm{g} / \mathrm{kg})$ & 12 & 7.5 & 15.5 \\
Wheat bran $(\mathrm{g} / \mathrm{kg})$ & 10 & 17.5 & 7 \\
Corn $(\mathrm{g} / \mathrm{kg})$ & 60 & 59 & 59 \\
Cotton cakes $(\mathrm{g} / \mathrm{kg})$ & 8 & 6.5 & 6 \\
Fish meal $(\mathrm{g} / \mathrm{kg})$ & 7 & 7 & 0.2 \\
Lysine $(\mathrm{g} / \mathrm{kg})$ & 0.2 & 0.2 & 0.2 \\
Methionine $(\mathrm{g} / \mathrm{kg})$ & 0.2 & 0.2 & 0.2 \\
Salt $(\mathrm{g} / \mathrm{kg})$ & 0.2 & 0.2 & 2.5 \\
Oyster shell $(\mathrm{g} / \mathrm{kg})$ & 2 & 1.5 & 0.25 \\
Premix $0.25(\mathrm{~g} / \mathrm{kg})$ & 0.25 & 0.25 & $\mathbf{1 0 0}$ \\
Total $(\mathrm{g} / \mathrm{kg})$ & $\mathbf{1 0 0}$ & $\mathbf{1 0 0}$ & 2800.0 \\
\hline Metabolisable energy $(\mathrm{kcal} / \mathrm{kg})$ & 2880.5 & 2969.6 & 20.1 \\
Crude protein $(\mathrm{g} / \mathrm{kg})$ & 18.6 & 17.2 & 0.92 \\
Lysine $(\mathrm{g} / \mathrm{kg})$ & 0.91 & 0.78 & 0.72 \\
Methionine + Cystine $(\mathrm{g} / \mathrm{kg})$ & 0.63 & 0.58 & 1.35 \\
Calcium $(\mathrm{g} / \mathrm{kg})$ & 1.11 & 0.91 & 0.35 \\
Digestible phosphate $(\mathrm{g} / \mathrm{kg})$ & 0.28 & 0.27 & \\
\hline
\end{tabular}


P.U. TOUGAN et al. / Int. J. Biol. Chem. Sci. 7(6): 2450-2467, 2013

Tableau 2: Correlations between technological and nutritional meat quality parameters in Holli (above diagonal) and North chickens (below diagonal).

\begin{tabular}{|c|c|c|c|c|c|c|c|c|c|c|c|c|c|c|c|c|}
\hline Variables & $\begin{array}{c}\text { Dry } \\
\text { Matter }\end{array}$ & Ash & Fat & Protein & $\begin{array}{l}\text { Shear } \\
\text { force }\end{array}$ & Extension & pH1 & pH4 & pH8 & pH12 & pH24 & $\mathbf{L}^{*}$ & $a^{*}$ & $\mathbf{b}^{*}$ & Hue & Chroma \\
\hline Dry Matter & 1 & $0.37 * *$ & $0.25^{\mathrm{NS}}$ & $0.57 * * *$ & $0.20^{\mathrm{NS}}$ & $0.09^{\mathrm{NS}}$ & $0.28 *$ & $0.27^{*}$ & $0.10^{\mathrm{NS}}$ & $0.11^{\mathrm{NS}}$ & $0.13^{\mathrm{NS}}$ & $-0.30^{*}$ & $0.35^{* *}$ & $0.34 * *$ & $-0.28^{*}$ & $0.38^{* *}$ \\
\hline Ash & $0.44 * *$ & 1 & $0.32 *$ & $-0.06^{\mathrm{NS}}$ & $0.31 *$ & $0.23^{\mathrm{NS}}$ & $0.02^{\mathrm{NS}}$ & $0.15^{\mathrm{NS}}$ & $0.08^{\mathrm{NS}}$ & $0.16^{\mathrm{NS}}$ & $0.27^{*}$ & $-0.27 *$ & $0.31^{*}$ & $0.28^{*}$ & $-0.15^{\mathrm{NS}}$ & $0.32 *$ \\
\hline Fat & $0.35^{*}$ & $0.05^{\mathrm{NS}}$ & 1 & $-0.64 * * *$ & $0.7 * * *$ & $0.6^{* * *}$ & $0.39 * *$ & $0.53 * * *$ & $0.3^{*}$ & $0.46^{* * * *}$ & $0.49 * * *$ & $-0.47 * * *$ & $0.85^{* * *}$ & $0.34 * *$ & $0.04^{\mathrm{NS}}$ & $0.8 * * *$ \\
\hline Protein & $0.29^{*}$ & $0.14^{\mathrm{NS}}$ & $-0.78 * * *$ & 1 & $-0.44^{* * * *}$ & $-0.41^{* *}$ & $-0.1^{\mathrm{NS}}$ & $-0.24^{\mathrm{NS}}$ & $-0.17^{\mathrm{NS}}$ & $-0.3^{*}$ & $-0.3^{*}$ & $0.16^{\mathrm{NS}}$ & $-0.45^{* * *}$ & $-0.04^{\mathrm{NS}}$ & $-0.24^{\mathrm{NS}}$ & $-0.4 * *$ \\
\hline Shear force & $-0.23^{\mathrm{NS}}$ & $-0.15^{\mathrm{NS}}$ & $0.54 * * *$ & $-0.69 * * *$ & 1 & $0.73 * * *$ & $0.25^{\mathrm{NS}}$ & $0.35^{* *}$ & $0.16^{\mathrm{NS}}$ & $0.33 *$ & $0.3 *$ & $-0.44 * * *$ & $0.67 * * *$ & $0.26^{\mathrm{NS}}$ & $0.13^{\mathrm{NS}}$ & $0.64 * * *$ \\
\hline Extension & $-0.32 *$ & $-0.30^{*}$ & $0.35^{*}$ & $-0.55 * * *$ & $0.75 * * *$ & 1 & $0.07^{\mathrm{NS}}$ & $0.23 \mathrm{NS}$ & $0.06^{\mathrm{NS}}$ & $0.22^{\mathrm{NS}}$ & $0.18^{\mathrm{NS}}$ & $-0.26^{\mathrm{NS}}$ & $0.5 * * *$ & $0.17^{\mathrm{NS}}$ & $0.1^{\mathrm{NS}}$ & $0.47 * * *$ \\
\hline pH1 & $0.09^{\mathrm{NS}}$ & $0.21^{\mathrm{NS}}$ & $0.36^{* *}$ & $-0.35^{*}$ & $0.25^{\mathrm{NS}}$ & $0.04^{\mathrm{NS}}$ & 1 & $0.76^{* * * *}$ & $0.46^{* * *}$ & $0.58^{* * *}$ & $0.44 * * *$ & $-0.27 *$ & $0.44 * * *$ & $0.14^{\mathrm{NS}}$ & $0.09^{\mathrm{NS}}$ & $0.43^{* * *}$ \\
\hline pH4 & $0.18^{\mathrm{NS}}$ & $0.18^{\mathrm{NS}}$ & $0.43 * *$ & $-0.34 *$ & $0.26^{\mathrm{NS}}$ & $0.07^{\mathrm{NS}}$ & $0.81^{* * *}$ & 1 & $0.69^{* * *}$ & $0.72^{* * *}$ & $0.55 * * *$ & $-0.37 * *$ & $0.54 * * *$ & $0.16^{\mathrm{NS}}$ & $-0.1^{\mathrm{NS}}$ & $0.52 * * *$ \\
\hline pH8 & $0.35^{*}$ & $0.25^{\mathrm{NS}}$ & $0.46^{* * *}$ & $-0.25^{\mathrm{NS}}$ & $0.22^{\mathrm{NS}}$ & $0.05^{\mathrm{NS}}$ & $0.72 * * *$ & $0.81 * * *$ & 1 & $0.66^{* * *}$ & $0.61 * * *$ & $-0.21^{\mathrm{NS}}$ & $0.15^{\mathrm{NS}}$ & $-0.19^{\mathrm{NS}}$ & $-0.1^{\mathrm{NS}}$ & $0.11^{*}$ \\
\hline pH12 & $0.22^{\mathrm{NS}}$ & $0.26^{\mathrm{NS}}$ & $0.35^{*}$ & $-0.24^{\mathrm{NS}}$ & $0.20^{\mathrm{NS}}$ & $0.03^{\mathrm{NS}}$ & $0.82 * * *$ & $0.76^{* * *}$ & $0.83 * * *$ & 1 & $0.86^{* * *}$ & $-0.49 * * *$ & $0.42 * *$ & $-0.08^{\mathrm{NS}}$ & $-0.03^{\mathrm{NS}}$ & $0.37 * *$ \\
\hline pH24 & $0.33^{*}$ & $0.33^{*}$ & $0.37 * *$ & $-0.19^{\mathrm{NS}}$ & $0.12^{\mathrm{NS}}$ & $-0.05^{\mathrm{NS}}$ & $0.73^{* * *}$ & $0.80^{* * * *}$ & $0.81 * * *$ & $0.83^{* * *}$ & 1 & $-0.43^{* * *}$ & $0.38 * *$ & $-0.15^{\mathrm{NS}}$ & $0.0004^{\mathrm{NS}}$ & $0.32^{*}$ \\
\hline $\mathbf{L}^{*}$ & $-0.47 * * *$ & $-0.25^{\mathrm{NS}}$ & $-0.54 * * *$ & $0.26^{\mathrm{NS}}$ & $-0.22^{\mathrm{NS}}$ & $-0.01^{\mathrm{NS}}$ & $-0.43^{* *}$ & $-0.44 * *$ & $-0.55^{* * *}$ & $-0.43 * *$ & $-0.46^{* * *}$ & 1 & $-0.56^{* * *}$ & $0.05^{\mathrm{NS}}$ & $-0.18^{\mathrm{NS}}$ & $-0.47 * * *$ \\
\hline$a^{*}$ & $0.39 * *$ & $0.16^{\mathrm{NS}}$ & $0.85^{* * *}$ & $-0.62 * * *$ & $0.45 * * *$ & $0.23^{\mathrm{NS}}$ & $0.34 *$ & $0.41 * *$ & $0.44 * *$ & $0.37 * *$ & $0.38^{* *}$ & $-0.69^{* * *}$ & 1 & $0.54^{* * * *}$ & $0.01^{\mathrm{NS}}$ & $0.98 * * *$ \\
\hline $\mathbf{b}^{*}$ & $0.21^{\mathrm{NS}}$ & $0.03^{\mathrm{NS}}$ & $0.25^{\mathrm{NS}}$ & $-0.13^{\mathrm{NS}}$ & $0.17^{\mathrm{NS}}$ & $0.12^{\mathrm{NS}}$ & $-0.10^{\mathrm{NS}}$ & $0.02^{\mathrm{NS}}$ & $-0.10^{\mathrm{NS}}$ & $-0.06^{\mathrm{NS}}$ & $-0.04^{\mathrm{NS}}$ & $-0.11^{\mathrm{NS}}$ & $0.43 * *$ & 1 & $-0.33^{*}$ & $0.67 * * *$ \\
\hline Hue & $0.15^{\mathrm{NS}}$ & $0.17^{\mathrm{NS}}$ & $0.54 * * *$ & $-0.46^{* * * *}$ & $0.23^{\mathrm{NS}}$ & $0.04^{\mathrm{NS}}$ & $0.37 * *$ & $0.41 * *$ & $0.50 * * *$ & $0.41 * *$ & $0.38 * *$ & $-0.59 * * *$ & $0.62 * * *$ & $-0.23^{\mathrm{NS}}$ & 1 & -0.07 \\
\hline Chroma & $0.40^{* *}$ & $0.15 \mathrm{NS}$ & $-0.78 * * *$ & $-0.55^{* * *}$ & $0.42 * *$ & $0.24^{\mathrm{NS}}$ & $0.26^{\mathrm{NS}}$ & $0.35^{\mathrm{NS}}$ & $0.35^{* *}$ & $0.29^{*}$ & $0.30 *$ & $-0.63 * * *$ & $0.97 * * *$ & $0.64 * * *$ & $0.47 * * *$ & 1 \\
\hline
\end{tabular}


P.U. TOUGAN et al. / Int. J. Biol. Chem. Sci. 7(6): 2450-2467, 2013

Tableau 3: Correlations between technological and nutritional meat quality parameters in Fulani (above diagonal) and Sahoue chickens (below diagonal).

\begin{tabular}{|c|c|c|c|c|c|c|c|c|c|c|c|c|c|c|c|c|}
\hline Variables & $\begin{array}{l}\text { Dry } \\
\text { Matter }\end{array}$ & Ash & Fat & Protein & $\begin{array}{l}\text { Shear } \\
\text { force }\end{array}$ & Extension & pH1 & pH4 & pH8 & pH12 & pH24 & $\mathbf{L}^{*}$ & $\mathbf{a}^{*}$ & b* & Hue & Chroma \\
\hline $\begin{array}{l}\text { Dry } \\
\text { Matter }\end{array}$ & 1 & $-0.07^{\mathrm{NS}}$ & $0.37 * *$ & $0.42 * *$ & $0.27^{\mathrm{NS}}$ & $0.25^{\mathrm{NS}}$ & $-0.13^{\mathrm{NS}}$ & $0.01^{\mathrm{NS}}$ & $-0.06^{\mathrm{NS}}$ & $-0.05^{\mathrm{NS}}$ & $-0.06^{\mathrm{NS}}$ & $-0.22^{\mathrm{NS}}$ & $0.35^{*}$ & $0.55^{* * *}$ & $-0.09^{\mathrm{NS}}$ & $0.43 * *$ \\
\hline Ash & $-0.07^{\mathrm{NS}}$ & 1 & $0.11^{\mathrm{NS}}$ & $-0.26^{\mathrm{NS}}$ & $-0.07^{\mathrm{NS}}$ & $-0.13^{\mathrm{NS}}$ & $0.004^{\mathrm{NS}}$ & $-0.3^{*}$ & $-0.33^{*}$ & $-0.29 *$ & $-0.29 *$ & $0.18^{\mathrm{NS}}$ & $0.13^{\mathrm{NS}}$ & $-0.05^{\mathrm{NS}}$ & $-0.01^{\mathrm{NS}}$ & $0.09^{\mathrm{NS}}$ \\
\hline Fat & $0.37 * *$ & $0.11^{\mathrm{NS}}$ & 1 & $-0.68 * * *$ & $0.65 * * *$ & $0.54 * * *$ & $0.30 *$ & $0.37 * *$ & $0.37 * *$ & $0.39 * *$ & $0.31^{*}$ & $-0.61 * * *$ & $0.81 * * *$ & $0.24^{\mathrm{NS}}$ & $0.39 * *$ & $0.76 * * *$ \\
\hline $\begin{array}{l}\text { Protein } \\
\text { Shear }\end{array}$ & $0.42 * *$ & $-0.26^{\mathrm{NS}}$ & $-0.68 * * *$ & 1 & $-0.42 * *$ & $-0.32 *$ & $-0.38 * *$ & $-0.30 *$ & $-0.35^{*}$ & $-0.38 * *$ & $-0.30 *$ & $0.41 * *$ & $-0.53 * * *$ & $0.19^{\mathrm{NS}}$ & $-0.45^{* * *}$ & $-0.42 * *$ \\
\hline force & $0.27^{\mathrm{NS}}$ & $-0.07^{\mathrm{NS}}$ & $0.65 * * *$ & $-0.42 * *$ & 1 & $0.73 * * *$ & $0.16^{\mathrm{NS}}$ & $0.40^{* *}$ & $0.44 * * *$ & $0.52 * * *$ & $0.46^{* * *}$ & $-0.79 * * *$ & $0.81 * * *$ & $0.34^{*}$ & $0.42 * *$ & $0.79 * * *$ \\
\hline Extension & $0.25^{\mathrm{NS}}$ & $-0.13^{\mathrm{NS}}$ & $0.54 * * *$ & $-0.32 *$ & $0.73^{* * *}$ & 1 & $-0.04^{\mathrm{NS}}$ & $0.27^{\mathrm{NS}}$ & $0.16^{\mathrm{NS}}$ & $0.17^{\mathrm{NS}}$ & $0.14^{\mathrm{NS}}$ & $-0.50 * * *$ & $0.55 * * *$ & $0.20^{\mathrm{NS}}$ & $0.35^{*}$ & $0.53^{* * *}$ \\
\hline pH1 & $-0.13^{\mathrm{NS}}$ & $0.004^{\mathrm{NS}}$ & $0.30^{*}$ & $-0.38^{* *}$ & $0.16^{\mathrm{NS}}$ & $-0.04^{\mathrm{NS}}$ & 1 & $0.49^{* * *}$ & $0.41^{* *}$ & $0.44 * * *$ & $0.33^{*}$ & $-0.33^{*}$ & $0.20^{\mathrm{NS}}$ & $-0.08^{\mathrm{NS}}$ & $0.21^{\mathrm{NS}}$ & $0.15^{\mathrm{NS}}$ \\
\hline pH4 & $0.01^{\mathrm{NS}}$ & $-0.3^{*}$ & $0.37 * *$ & $-0.30^{*}$ & $0.40^{* *}$ & $0.27^{\mathrm{NS}}$ & $0.49^{* * *}$ & 1 & $0.77 * * *$ & $0.6^{* * *}$ & $0.60 * * *$ & $-0.42 * *$ & $0.28^{*}$ & $0.12^{\mathrm{NS}}$ & $0.17^{\mathrm{NS}}$ & $0.28^{*}$ \\
\hline pH8 & $-0.06^{\mathrm{NS}}$ & $-0.33^{*}$ & $0.37 * *$ & $-0.35^{*}$ & $0.44 * * *$ & $0.16^{\mathrm{NS}}$ & $0.41 * *$ & $0.77 * * *$ & 1 & $0.80 * * *$ & $0.81 * * *$ & $-0.63 * * *$ & $0.37^{* *}$ & $0.03^{\mathrm{NS}}$ & $0.31^{*}$ & $0.34^{*}$ \\
\hline $\begin{array}{l}\text { pH12 } \\
\text { pH24 }\end{array}$ & $\begin{array}{l}-0.05^{\mathrm{NS}} \\
-0.06^{\mathrm{NS}}\end{array}$ & $\begin{array}{l}-0.29^{*} \\
-0.29^{*}\end{array}$ & $\begin{array}{l}0.39 * * \\
0.31 *\end{array}$ & $\begin{array}{l}-0.38 * * \\
-0.30 *\end{array}$ & $\begin{array}{l}0.52^{* * * *} \\
0.46^{* * * *}\end{array}$ & $\begin{array}{l}0.17^{\mathrm{NS}} \\
0.14^{\mathrm{NS}}\end{array}$ & $\begin{array}{l}0.44^{* * *} \\
0.33^{*}\end{array}$ & $\begin{array}{l}0.6^{* * * *} \\
0.60^{* * * *}\end{array}$ & $\begin{array}{l}0.80^{* * * *} \\
0.81 * * *\end{array}$ & $\begin{array}{l}1 \\
0.79 * * *\end{array}$ & $\begin{array}{l}0.79 * * * \\
1\end{array}$ & $\begin{array}{l}-0.67 * * * \\
-0.58^{* * * *}\end{array}$ & $\begin{array}{l}0.43^{* *} \\
0.34^{*}\end{array}$ & $\begin{array}{l}0.02^{\mathrm{NS}} \\
0.04^{\mathrm{NS}}\end{array}$ & $\begin{array}{l}0.32^{*} \\
0.26^{\mathrm{NS}}\end{array}$ & $\begin{array}{l}0.38^{* * *} \\
0.32^{*}\end{array}$ \\
\hline $\mathbf{L}^{*}$ & $-0.22^{\mathrm{NS}}$ & $0.18^{\mathrm{NS}}$ & $-0.61 * * *$ & $0.41^{* *}$ & $-0.79 * * *$ & $-0.50^{* * *}$ & $-0.33^{*}$ & $-0.42^{* *}$ & $-0.63^{* * *}$ & $-0.67 * * *$ & $-0.58^{* * *}$ & 1 & $-0.77 * * *$ & $-0.23^{\mathrm{NS}}$ & $-0.52^{* * * *}$ & $-0.73 * * *$ \\
\hline$a^{*}$ & $0.35^{*}$ & $0.13^{\mathrm{NS}}$ & $0.81^{* * * *}$ & $-0.53 * * *$ & $0.81 * * *$ & $0.55^{* * * *}$ & $0.20^{\mathrm{NS}}$ & $0.28^{*}$ & $0.37 * *$ & $0.43^{* *}$ & $0.34^{*}$ & $-0.77^{* * *}$ & 1 & $0.46^{* * *}$ & $0.46^{* * * *}$ & $0.98 * * *$ \\
\hline$b^{*}$ & $0.55^{* * * *}$ & $-0.05^{\mathrm{NS}}$ & $0.24^{\mathrm{NS}}$ & $0.19^{\mathrm{NS}}$ & $0.34 *$ & $0.20^{\mathrm{NS}}$ & $-0.08^{\mathrm{NS}}$ & $0.12^{\mathrm{NS}}$ & $0.03^{\mathrm{NS}}$ & $0.02^{\mathrm{NS}}$ & $0.04^{\mathrm{NS}}$ & $-0.23^{\mathrm{NS}}$ & $0.46^{* * *}$ & 1 & $-0.46 * * *$ & $0.61 * * *$ \\
\hline $\begin{array}{l}\text { Hue } \\
\text { Chroma }\end{array}$ & $\begin{array}{l}-0.09^{\mathrm{NS}} \\
0.43^{* *}\end{array}$ & $\begin{array}{l}-0.01^{\mathrm{NS}} \\
0.09^{\mathrm{NS}}\end{array}$ & $\begin{array}{l}0.39^{* *} \\
0.76^{* * * *}\end{array}$ & $\begin{array}{l}-0.45^{* * *} \\
-0.42^{* * *}\end{array}$ & $\begin{array}{l}0.42 * * \\
0.79 * * *\end{array}$ & $\begin{array}{l}0.35^{*} \\
0.53 * * *\end{array}$ & $\begin{array}{l}0.21^{\mathrm{NS}} \\
0.15^{\mathrm{NS}}\end{array}$ & $\begin{array}{l}0.17^{\mathrm{NS}} \\
0.28^{*}\end{array}$ & $\begin{array}{l}0.31 * \\
0.34 *\end{array}$ & $\begin{array}{l}0.32 * \\
0.38 * *\end{array}$ & $\begin{array}{l}0.26^{\mathrm{NS}} \\
0.32 *\end{array}$ & $\begin{array}{l}-0.52 * * * \\
-0.73 * * *\end{array}$ & $\begin{array}{l}0.46^{* * * *} \\
0.98 * * *\end{array}$ & $\begin{array}{l}-0.46^{* * *} \\
0.61 * * *\end{array}$ & $\begin{array}{l}1 \\
0.33^{*}\end{array}$ & $0.33^{*}$ \\
\hline
\end{tabular}


P.U. TOUGAN et al. / Int. J. Biol. Chem. Sci. 7(6): 2450-2467, 2013

Tableau 4: Correlations between technological and nutritional meat quality parameters in South chicken.

\begin{tabular}{|c|c|c|c|c|c|c|c|c|c|c|c|c|c|c|c|c|}
\hline & Dry & & & & Shear & & & & & & & & & & & \\
\hline Variables & Matter & Ash & Fat & Protein & force & Extension & pH1 & pH4 & pH8 & pH12 & pH24 & $\mathbf{L}^{*}$ & $a^{*}$ & $\mathbf{b}^{*}$ & Hue & Chroma \\
\hline Dry Matter & 1 & & & & & & & & & & & & & & & \\
\hline Ash & $0.03^{\mathrm{NS}}$ & 1 & & & & & & & & & & & & & & \\
\hline Fat & $0.28 *$ & $-0.02^{\mathrm{NS}}$ & 1 & & & & & & & & & & & & & \\
\hline Protein & $0.44 * * *$ & $-0.05^{\mathrm{NS}}$ & $-0.73 * * *$ & 1 & & & & & & & & & & & & \\
\hline Shear force & $-0.06^{\mathrm{NS}}$ & $0.01^{\mathrm{NS}}$ & $0.55^{* * * *}$ & $-0.56 * * *$ & 1 & & & & & & & & & & & \\
\hline Extension & $0.27^{\mathrm{NS}}$ & $-0.06^{\mathrm{NS}}$ & $0.35 *$ & $-0.14^{\mathrm{NS}}$ & $0.33^{*}$ & 1 & & & & & & & & & & \\
\hline pH1 & $0.08^{\mathrm{NS}}$ & $0.10^{\mathrm{NS}}$ & $0.36 * *$ & $-0.29 *$ & $0.38 * *$ & $0.01^{\mathrm{NS}}$ & 1 & & & & & & & & & \\
\hline pH4 & $0.21^{\mathrm{NS}}$ & $0.13^{\mathrm{NS}}$ & $0.27 *$ & $-0.12^{\mathrm{NS}}$ & $0.32 *$ & $0.09^{\mathrm{NS}}$ & $0.78^{* * *}$ & 1 & & & & & & & & \\
\hline pH8 & $0.17^{\mathrm{NS}}$ & $0.12^{\mathrm{NS}}$ & $0.34 *$ & $-0.22^{\mathrm{NS}}$ & $0.29 *$ & $0.09^{\mathrm{NS}}$ & $0.74 * * *$ & $0.89 * * *$ & 1 & & & & & & & \\
\hline
\end{tabular}


P.U. TOUGAN et al. / Int. J. Biol. Chem. Sci. 7(6): 2450-2467, 2013

\begin{tabular}{|c|c|c|c|c|c|c|c|c|c|c|c|c|c|c|c|c|}
\hline pH12 & $0.14^{\mathrm{NS}}$ & $0.01^{\mathrm{NS}}$ & $0.24 *$ & $-0.12^{\mathrm{NS}}$ & $0.25^{\mathrm{NS}}$ & $0.11^{\mathrm{NS}}$ & $0.67^{* * * *}$ & $0.77^{* * * *}$ & $0.79^{* * * *}$ & 1 & & & & & & \\
\hline pH24 & $0.17^{\mathrm{NS}}$ & $0.13^{\mathrm{NS}}$ & $0.39 * *$ & $-0.25^{\mathrm{NS}}$ & $0.32 *$ & $0.05^{\mathrm{NS}}$ & $0.61 * * *$ & $0.68 * * *$ & $0.56^{* * *}$ & $0.67 * * *$ & 1 & & & & & \\
\hline $\mathbf{L}^{*}$ & $-0.24^{\mathrm{NS}}$ & $-0.15^{\mathrm{NS}}$ & $-0.73 * * *$ & $0.53 * * *$ & $-0.42 * *$ & $-0.31 *$ & $-0.53 * * *$ & $-0.50 * * *$ & $-0.53 * * *$ & $-0.38 * *$ & $-0.51 * * *$ & 1 & & & & \\
\hline$a^{*}$ & $0.32 *$ & $0.11^{\mathrm{NS}}$ & $0.87 * * *$ & $-0.60 * * *$ & $0.59 * * *$ & $0.35^{*}$ & $0.47^{* * *}$ & $0.42 * *$ & $0.44 * *$ & $0.30^{*}$ & $0.53^{* * * *}$ & $-0.76 * * *$ & 1 & & & \\
\hline$b^{*}$ & $0.37 * *$ & $-0.07^{\mathrm{NS}}$ & $0.18^{\mathrm{NS}}$ & $0.10^{\mathrm{NS}}$ & $-0.00^{\mathrm{NS}}$ & $0.14^{\mathrm{NS}}$ & $-0.005^{\mathrm{NS}}$ & $0.10^{\mathrm{NS}}$ & $0.14^{\mathrm{NS}}$ & $0.07^{\mathrm{NS}}$ & $-0.02^{\mathrm{NS}}$ & $0.01^{\mathrm{NS}}$ & $0.34 *$ & 1 & & \\
\hline Hue & $0.11^{\mathrm{NS}}$ & $-0.05^{\mathrm{NS}}$ & $0.38 * *$ & $-0.27^{\mathrm{NS}}$ & $0.30 *$ & $0.30 *$ & $0.11^{\mathrm{NS}}$ & $0.12^{\mathrm{NS}}$ & $0.10^{\mathrm{NS}}$ & $0.07^{\mathrm{NS}}$ & $0.31 *$ & $-0.36 * *$ & $0.43^{* *}$ & $-0.12^{\mathrm{NS}}$ & 1 & \\
\hline Chroma & $0.37 * *$ & $0.01^{\mathrm{NS}}$ & $0.82^{* * * *}$ & $-0.52 * * *$ & $0.53 * * *$ & $0.53^{* * * *}$ & $0.42 * *$ & $0.39^{* * *}$ & $0.42 * *$ & $0.27 *$ & $0.48^{* * * *}$ & $-0.68 * * *$ & $0.98^{* * * *}$ & $0.52 * * *$ & $0.37 * *$ & 1 \\
\hline
\end{tabular}

NS: Non significant; *: $\mathrm{P}<0.05 ;$ **: $\mathrm{P}<0.01 ; * * *: \mathrm{P}<0.001 ; ; \mathrm{pHi}: \mathrm{pH}$ recorded i hours post mortem ; $\mathrm{L}^{*}$ : Luminance; $\mathrm{a}^{*}$ : redness; $\mathrm{b}^{*}$ : yellowness... 


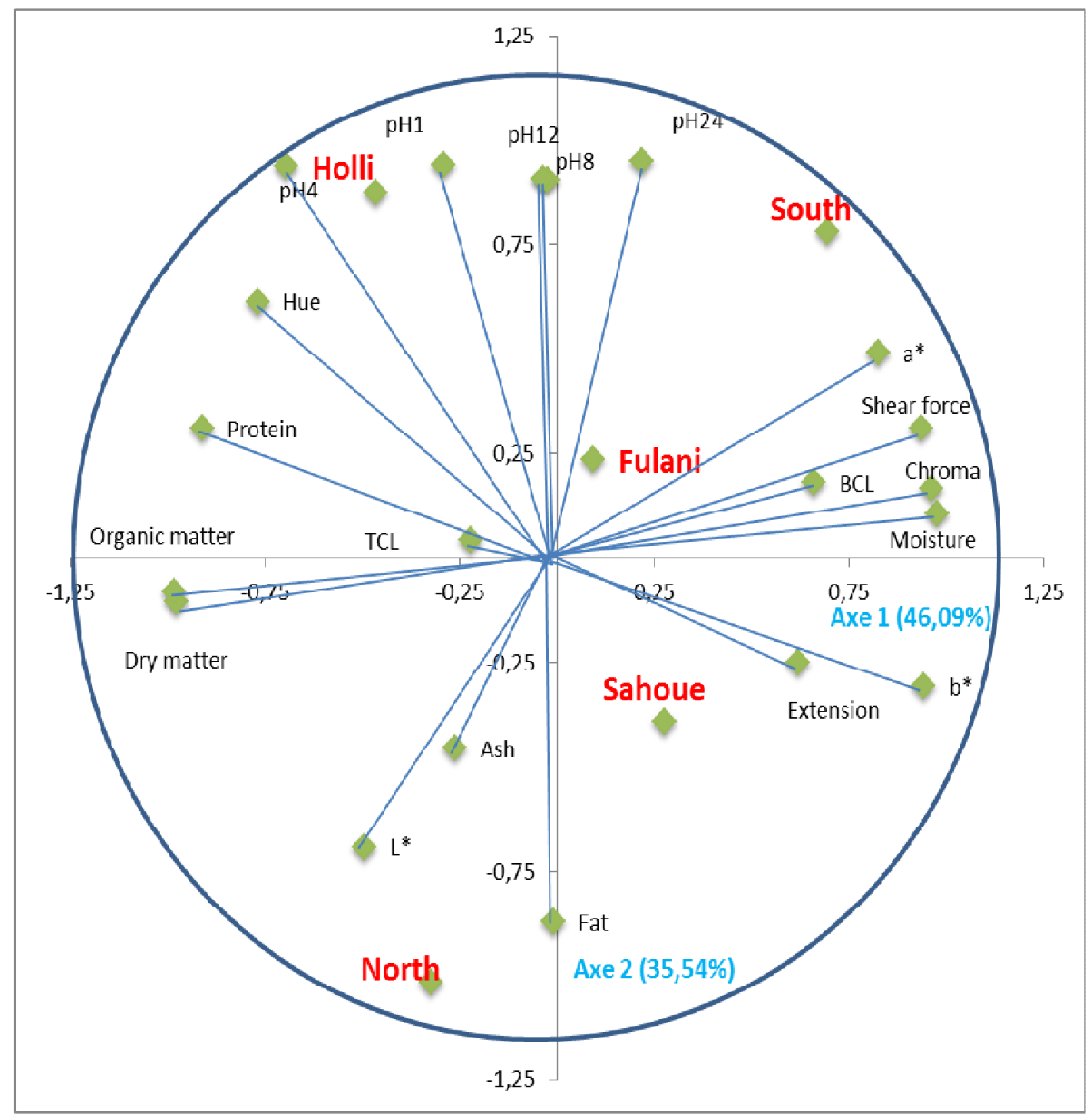

Figure 1: Principal Components Analysis (PCA) of indigenous chicken technological and nutritional meat quality parameters of Holli, Fulani, Sahoue, North and South ecotypes of Benin ; TCL: Thigh-drumstick cooking loss; BCL: Breast Cooking loss; ; ; pHi : pH recorded i hours post mortem ; L*: Luminance; $\mathrm{a}^{*}$ : redness; $\mathrm{b}^{*}$ : yellowness..

\section{DISCUSSION}

\section{Correlations between technological and nutritional meat quality parameters}

The correlations between technological and nutritional meat quality parameters obtained herein differ among chicken genetic types. Technological meat quality parameters were more associated to nutritional meat quality traits in Fulani and Holli, chickens than Sahoue, South and North chickens. The observed differences in the relationships between meat qualities traits among genotype could be related to the genetic variability of carcass traits that exist among those studied 
ecotypes (Tougan et al., 2013a). This result confirms the finding of Youssao et al. (2010) who showed that indigenous chicken populations of Benin are characterized by a great genetic diversity than that reported for commercial lines chicken (Granevitze et al., 2007; Muchadeyi et al., 2007; Berthouly et al., 2008). In accordance with Havenstein et al. (2003a, 2003b), technological and nutritional meat quality depend on genotype. Franco et al. $(2012,2013)$ also found great difference in meat quality traits between Mos and Sasso T44 roosters.

Overall, dry matter content was positively correlated with protein content, redness $\left(a^{*}\right)$ chroma value and to a lesser extent fat content, but negatively correlated with the luminance $\left(\mathrm{L}^{*}\right)$. This result indicated that an increase in dry matter content led to significant increases in protein, redness $\left(a^{*}\right)$ chroma value and to a lesser extent fat content, but to significant decrease in meat lightness. According to Oluwatosin et al. (2007), an increase in the level of dry matter led to significant increases in organic matter and ether extract, suggesting that an increase in dry matter content would result to toughness of muscles rather than to higher carcass quality. This difference between the results obtained herein and those of Oluwatosin et al. (2007) in the relationship between dry matter content and fat content could be explained by the lower fat content recorded in the local chickens of Benin (1.83$2.09 \%$ of raw meat) comparatively to the fat content (4.75 to $6.44 \%$ of raw meat) of chicken strains used by Oluwatosin et al. (2007), and the genetic variability of those chicken breeds.

However, Oluwatosin et al. (2007) found that as the moisture content in the cockerels' muscles increased, significant $(\mathrm{P}<0.05)$ increases in crude protein and ash content occurred in disagreement with the current results. This difference in relationships between moisture content and protein content could be due to the weak slaughter weight and lower fat content in indigenous chicken meat of Benin compared to those of 28-weeks old cockerels of Nera, Bovan, Harco and Nigerian local strains used by Oluwatosin et al. (2007).

Fat content was more associated with the other meat quality traits in chicken of all ecotypes studied herein. This finding corroborates the results of Chabault et al. (2012) who reported positive genetic correlation between intramuscular fat content and luminance $\left(r_{g}=0.42\right)$ as well as the shear force $\left(r_{g}=0.57\right)$ in free range naked-neck chickens, a slow-growing line selected by the SASSO breeding company. Previous studies had reported positive genetic correlations of abdominal fat weight and abdominal fat percentage with carcass weight in chickens (Deeb and Lamont, 2002; Musa et al., 2006). Furthermore, except dry matter content, ash content, luminance and yellowness, protein content was negatively correlated with the others technological and nutritional meat quality parameters with the high negative correlations found with the shear force and redness in all genetic types studied in the present work. As found herein, Chabault et al. (2012) found strong negative genetic correlations between the ultimate $\mathrm{pH}$ and the lightness, yellowness and drip loss of the meat. Similarly, Fletcher (1999) had reported high negatives correlations between $\mathrm{pH}$ and lightness $(\mathrm{P}<0.001 ; \mathrm{r}=-0.636)$ and yellowness $(\mathrm{P}<0.01 ; \mathrm{r}=-0.2)$. These results are consistent with those previously reported for turkey breast meat (Barbut, 1993). In the same way, Berri et al. (2007) found high significant negative correlation between the ultimate $\mathrm{pH}$ and the lightness $(\mathrm{P}<0.001$; -0.61). In short, when $\mathrm{pH}$ decreases, lightness, yellowness and drip loss of the meat increase. However, Contreras-Castillo et al. 
(2007) found that correlation between $\mathrm{L}^{*}$ and $\mathrm{pH}$ values was not significant in male Cobb broilers.

In the present study, the values for $\mathrm{a}^{*}$ were inversely related to the $\mathrm{L}^{*}$ values in all ecotypes used suggesting that the higher the level of lightness, the lower the redness of the chicken meat. This finding is consistent with those of Askit et al. (2006) in Ross 308 broiler, Contreras-Castillo et al. (2007) in male Cobb broilers, and de Jesus Silva (2011) in broiler meat. According to Le Bihan-Duval et al. (2001), the redness and yellowness of chicken are linked, such that meat with higher redness tends to present higher levels of yellowness, similar to the association found in this study.

Contrary to the findings of ContrerasCastillo et al. (2007) in male Cobb broilers who pointed out that the correlation between $\mathrm{L}^{*}$ and $\mathrm{pH}$ values was not significant, luminance was highly and negatively associated to $\mathrm{pH} 8$ and $\mathrm{pH} 24$, moderately and negatively correlated with $\mathrm{pH} 1, \mathrm{pH} 4$ and pH12 in the current study. Our results were consistent with the reports of Askit et al. (2006) in Ross 308 broiler and Chabault et al. (2012) in free range naked-neck chickens. Franco et al. (2013) also found a negative correlation $(r=-0.38 ; p<0.01)$ between $L^{*}$ and $\mathrm{pH}$ was found in Sasso T-44 line and Mos breed chickens. Similarly, this finding is in accordance with that reported by Le BihanDuval et al. (2008) and de Jesus Silva (2011). These significant inverse correlations found between $\mathrm{L}^{*}$ and $\mathrm{pH} 24$ suggests that the meat of the studied ecotypes tends to present greater lightness when the $\mathrm{pH}$ is smaller, indicating the occurrence of protein denaturation 24 hours after slaughter. This is in agreement with the results of Le BihanDuval et al. (2003), who reported that the greater the degree of protein denaturation, the less the amount of light transmitted through the fibers and the more light that is dispersed, which makes the meat pale. Debut et al. (2003) also found negative and moderated correlations between the lightness of the meat and the $\mathrm{pH} 24$ hours after slaughter.

Non-significant correlations or to a leaser extend weak and positive relationships were found between shear force and $\mathrm{pH}$ values in the current study indicating that meat with a low $\mathrm{pH}$ is less tender. This observation is in disagreement with the finding of Barbut (1993) and ContrerasCastillo et al. (2007) in broiler who found that shear values were highly inversely correlated with $\mathrm{pH}$. These discrepancies in relationships between shear force and $\mathrm{pH}$ values could be related to the difference in genotypes of chickens used and their slaughter age. Indigenous chicken populations of Benin are of slow-growing type slaughtered at 24 weeks old, while Cobb broilers used by ContrerasCastillo et al. (2007) are heavier and fastgrowing chicken line slaughtered at 6 weeks old. According to Anadón (2002), the texture of the meat is closely related to the amount of intramuscular water and, thus, inversely associated to the dry matter content of the meat, similar to the correlations found herein.

Overall, the obtained results showed that all the meat quality traits measured were good indicators of fat content.

\section{Principal components analysis of technological and nutritional meat quality parameters}

Principal components analysis of technological and nutritional meat quality parameters discriminated the five ecotypes according to their meat quality. Similar principal components analysis results were reported by Tougan et al. (2013d) when studying relationships between carcass traits and offal components in local poultry of Benin. This finding confirms that there is a 
gene linkage effect operating on technological and nutritional meat quality as found by several authors in carcass quality traits in chicken (Muhiuddin 1993; Olawumi 2013).

South chickens and to a lesser extent Fulani and Sahoue chickens were characterized by higher moisture, shear force, breast cooking loss, yellowness, extension and pH24, while Holli chickens were characterized by greater protein content, organic matter content, dry matter, thigh cooking loss, hue value, $\mathrm{pH} 1, \mathrm{pH} 4, \mathrm{pH} 8$ and pH12. North chickens were characterized by higher ash content, fat content and luminance. This variability in technological and nutritional meat quality characteristics among birds may be related to the genetic variability of chickens used in the present study since they were reared under the same breeding system and environmental conditions. These findings corroborate the observations of Tougan et al. (2013d) on the principal components analysis of carcass characteristics and offal component of the same indigenous chicken ecotypes of Benin. In the same way, Debut et al. (2003) showed that principal components analysis (PCA) of meat quality traits of slow-growing and fast growing strains of chicken discriminated both genotypes according to their meat quality. These authors showed that PCA opposed slow-growing and fast growing chickens with the slow-growing chickens characterized by higher thigh $\mathrm{pHu}$, breast redness, thigh technological yield, breast $\mathrm{pHu}$, thigh $\mathrm{pH} 15$ and thigh redness, while fast growing chickens were characterized by higher breast water loss, breast yellowness, breast lightness, thigh lightness, thigh yellowness, thigh redness and breast $\mathrm{pH} 15$.

\section{Conclusion}

The current work on phenotypic correlations between technological and nutritional meat quality parameters in indigenous chicken populations of Benin showed that fat content was the most associated with the other meat quality traits in chicken of all ecotypes studied. Furthermore, technological meat quality parameters were more associated to nutritional meat quality parameters in Fulani and Holli, chickens than Sahoue, South and North chickens. The principal components analysis of technological and nutritional meat quality parameters discriminated the five ecotypes according to their meat quality properties. South chickens and to a lesser extent Fulani and Sahoue chickens were characterized by higher dry matter, shear force, breast cooking loss, yellowness, extension and $\mathrm{pH} 24$, while Holli chickens were characterized by greater protein content, organic matter content, moisture, thigh cooking loss, hue value, $\mathrm{pH} 1$, pH4, pH8 and pH12. North chickens were characterized by higher ash content, fat content and luminance. Therefore, improve one meat quality trait will improve the other traits as a correlated response.

\section{ACKNOWLEDGEMENTS}

The authors thank the "Commission Universitaire pour le Developpement" and UAC01 Activity for their financial contribution.

\section{REFERENCES}

Aksit M, Yalcın S, O“zkan S, Metin K, O“zdemir D. 2006. Effects of temperature during rearing and crating on stress parameters and meat quality of broilers. Poult. Sci., 85: 1867-1874.

Anadón HLS. 2002. Biological, nutritional and processing factors affecting breast meat quality of broilers. These (Doctor of Philosophy in Animal and Poultry Sciences); Virginia Polytechnic Institute and State University. 181p. 
http://scholar.lib.vt.edu/theses/available/e td-02212002-113821/unrestricted/

Dissertation.pdf.

Barbut S. 1993. Colour measurements for evaluating the pale soft exudative (PSE) occurrence in turkey meat. Food Res. Int., 26: 39-43.

Berri C, Le Bihan-Duval E, Debut M, SantéLhoutellier V, Baéza E, Gigaud V, Jégo Y, Duclos MJ. 2007. Consequence of muscle hypertrophy on characteristics of Pectoralis major muscle and breast meat quality of broiler chickens. J. Anim. Sci., 85: 2005-2011.

Berthouly C, Bed'Hom B, Tixier-Boichard M, Chen CF, Lee YP, Laloë D, Legros H, Verrier E, Rognon X. 2008. Using molecular and multivariate methods to study the genetic diversity on local European and Asian chicken breeds. Anim. Genet., 39: 121-129.

Chabault M., Baéza E, Gigaud V, Chartrin P, Chapuis H, Boulay M, Arnould C, D'Abbadie F, Berri C and Le BihanDuval E. 2012. Analysis of a slowgrowing line reveals wide genetic variability of carcass and meat qualityrelated traits. BMC Genetics, 8p. http://www.biomedcentral.com/14712156/13/90.

Contreras-Castillo C, Pinto AA, Souza GL, Beraquet NJ, Aguiar AP, Cipolli KMV, Mendes CMI, Ortega EM. 2007. Effects of Feed Withdrawal Periods on Carcass Yield and Breast Meat Quality of Chickens Reared Using an Alternative System. J. Appl. Poult. Res., 16: 613622.

CountryStat/Benin. 2012. Base de données statistiques, consulté à l'adresse, http://countrystat.org/ben ou http://www. fao.org/economic/ess/countrystat/en/.
De Jesus Silva LB. 2011. Phenotypic correlations among meat quality traits in broilers. Cienc. Rural, 41(8): 11p.

Debut M, Berri C, Baéza E, Sellier N, Arnould C, Guéméné D, Jehl N, Boutten B, Beaumont C, Le Bihan-Duval E. 2003. Variation of chicken technological meat quality in relation to genotype and preslaughter stress conditions. Poult. Sci, 82: $1829-1838$.

Deeb N, Lamont SJ. 2002. Genetic architecture of growth and body composition in unique chicken populations. Heredity, 93: 107-118.

Fletcher DL. 1999. Broiler Breast Meat Color Variation, pH, and Texture. Poult. Sci., 78: 1323-1327.

Franco D, Rois D, Vazquez JA, LorenzoRodriguez JM. 2013. Carcass morphology and meat quality from roosters slaughtered at eight months affected by genotype and finishing feeding. Spanish J. Agric. Res., 11(2). doi: 10.5424/sjar/2013112-3094.

Granevitze Z, Hillel J, Chen GH, Cuc NTK, Feldam M, Eding H, Weigend S. 2007. Genetic diversity within chicken populations from different continents and management histories. Anim. Genet., 38: 576-583.

Havenstein GB, Ferket PR, Qureshi MA. 2003a. Growth, livability, and feed conversion of 1957 versus 2001 broilers when fed representative 1957 and 2001 broiler diets. Poult. Sci., 82: 1500-1508.

Havenstein GB, Ferket PR, Qureshi MA. 2003b. Carcass composition and yield of 1957 versus 2001 broilers when fed representative 1957 and 2001 broiler diets. Poult. Sci., 82: 1509-1518.

Houessionon FJB. 2011. Typologie des élevages de poulets locaux de l'espèce Gallus gallus en aviculture familiale au Bénin, Mémoire de Master en Production 
et Santé Animales, Ecole Polytechnique d'Abomey-Calavi, Université d'AbomeyCalavi, 98p.

INSAE (Institut National de Statistique et l'Analyse Economique). 2010. Rapport annuel d'activités. INSAE : Cotonou, Bénin, 360p.

Le Bihan-Duval E, Debut M, Berri C, Sellier N, Santé-L'houtellier V, Jégo Y, Beaumont C. 2008. Chicken meat quality: genetic variability and relationship with growth and muscle characteristics. BMC Genet., 9: 53p.

Le Bihan-Duval E, Berri C, Baéza E, Millet N, Beaumont C. 2001. Estimation of the genetic parameters of meat characteristics and their genetic correlations with grow and body composition in an experimental broiler line. Poult. Sci., 80(7) : 839-843.

Le Bihan-Duval E, Berri C, Baéza E, Santé V, Astruc T, Rémignon H, Le Pottier G, Bentley J, Beaumont C, Fernandez X. 2003. Genetic parameters of meat technological quality traits in a grandparental commercial line of turkey. Genet. Select. Ev., 35: 623-635.

Mankor A. 2009. Evolution du secteur de l'élevage Ouest africain, Consommation urbaine de viande en Afrique de l'Ouest : l'exemple de Dakar. Grain de Sel, 46-47: 16-17.

Muchadeyi FC, Eding H, Wollny CBA, Groeneveld E, Makusa SM, Shamseldin R, Simianer H, Weigend S. 2007. Absence of population substructuring in Zimbabwe chicken ecotypes inferred using microsatellites analysis. Anim. Genet., 38: 332-339.

Muhiuddin G. 1993. Estimates of genetic and phenotypic parameters of some performance traits in beef cattle. Anim. Breed., 66: 495 - 522.

Musa HH, Chen GH, Cheng JH, Li BC, Mekki DM. 2006. Study on carcass characteristics of chicken breeds raised under the intensive condition. Int. J. Poult. Sci., 5(6): 530-533.

Olawumi SO. 2013. Phenotypic correlations between live body weight and carcass traits in arbor acre breed of broiler chicken. Int. J. Sci. Nat., 4(1): 145-149.

Oluwatosin O, Olawoyin E, Agiang A, De Neji C, Iso E. 2007. Nutritional evaluation of the thigh and breast muscles of four cockerel strains. Afr. J. Anim. Biomed. Sci., 2(2): 26-31.

SAS. 2006. SAS/STAT User's Guide, vers, 6, 4th edn. SAS Inst: Cary, NC, USA.

Sonaiya EB, Swan SEJ. 2004. Production en Aviculture Familiale, un Manuel Technique. Organisation des NationsUnies pour l'Alimentation et l'Agriculture: Rome; 140p

Tougan PU, Youssao AKI, Dahouda M, Salifou CFA, Ahounou GS, Kpodekon M, Mensah GA, Kossou DN, Amenou C, Kogbeto C, Thewis A. 2013a. Variability of carcass traits of local poultry populations of Gallus gallus species of Benin by genetic type, breeding mode and slaughter age. Int. J. Poult. Sci., (in press).

Tougan PU, Dahouda M, Ahounou GS, Salifou CFA, Kpodekon MT, Mensah GA, Kossou DNF, Amenou C, Kogbeto CE, Thewis A, Youssao IAK. 2013b. Effect of breeding mode, type of muscle and slaughter age on technological meat quality of local poultry population of Gallus gallus species of Benin. Int. J. Biosci., 3(6):1-17.

Tougan PU, Dahouda M, Salifou CFA, Ahounou GS, Kpodekon MT, Mensah GA, Kossou DNF, Amenou C, Kogbeto CE, Lognay G, Thewis A, Youssao IAK. 2013d. Assessment of nutritional quality of meat of local poultry population of 
Gallus gallus specie of Benin. J. Anim. Plant Sci., (in press).

Tougan UP. 2008. Enquête sur les systèmes d'élevage et le polymorphisme moléculaire des populations locales de volaille de l'espèce Gallus gallus au Nord et au Sud du Bénin. Mémoire DIT, Ecole Polytechnique d'Abomey-Calavi, Université d'Abomey-Calavi, Benin 125 p.

Youssao AKI, Adehan R, Kpodekon M, Bonou G, Dougnon J, Koutinhouin B. 2007. Diversité génétique des populations locales de volailles de l'espèce Gallus gallus au Sud et au Nord du Bénin. 1er Colloque de l'UAC des Sciences, Cultures et Technologies, du 25 au 29 juin 2007 à Abomey-Calavi, 230-234.

Youssao AKI, Assogba NM, Alkoiret T, Dahouda M, Idrissou N-D, Kayang BB, Yapi-Gnaoré V, Assogba HM, Houinsou AS, Ahounou S, Tougan UP, Rognon X, Tixier-Boichard M. 2012. Comparison of growth performance, carcass characteristics and sensory characters of
Benin indigenous chickens and Label Rouge (T55×SA51). Afr. J. Biotech., 6: 15569-15579.

Youssao AKI, Senou M, Dahouda M, Kpodekon TM, Jenontin J, Idrissou N-D, Bonou AG, Tougan PU, Assogba HM, Ankole E, Rognon X, Tixier-Boichard M. 2009. Genetic improvement of local chickens by crossing with the Label Rouge (T55XSA51): growth performances and heterosis effects. Int. J. Poult. Sci., 8(6): 536-544.

Youssao AKI, Tobada PC, Koutinhoun BG, Dahouda M, Idrissou N-D, Bonou GA, Tougan UP, Ahounou S, Yapi-Gnaoré V, Kayang B, Rognon K, Tixier-Boichard M. 2010. Phenotypic characterization and molecular polymorphism of indigenous poultry populations of the species Gallus gallus of Savannah and Forest ecotypes of Benin. Afr. J. Biotech., 9(3): 369-381. 